\title{
Robust Adaptive Generalized Projective Synchronization of Chaotic Systems with Uncertain Disturbances
}

\author{
Zhen Jia ${ }^{1,2}$ and Guangming Deng ${ }^{1}$ \\ ${ }^{1}$ College of Science, Guilin University of Technology, Guilin 541004, China \\ ${ }^{2}$ Guangxi Key Laboratory of Spatial Information and Geomatics, Guilin 541004, China \\ Correspondence should be addressed to Zhen Jia, jjjzzz0@163.com
}

Received 27 July 2012; Revised 16 September 2012; Accepted 19 September 2012

Academic Editor: Nazim Idrisoglu Mahmudov

Copyright $\odot 2012$ Z. Jia and G. Deng. This is an open access article distributed under the Creative Commons Attribution License, which permits unrestricted use, distribution, and reproduction in any medium, provided the original work is properly cited.

\begin{abstract}
The generalized projective synchronization (GPS) of chaotic systems with uncertain parameter noise and external disturbance is discussed. Based on the adaptive technique, a response system is constructed, and a novel adaptive controller is designed to guarantee the GPS between the driveresponse systems, and to eliminate the effect of external disturbance and parameters noise on GPS. The conclusion is proved theoretically, and corresponding numerical simulations are provided to verify the effectiveness of the proposed method.
\end{abstract}

\section{Introduction}

The concept of chaos synchronization was introduced in the first time by Pecora and Carroll and an effective synchronization method was proposed in 1990 [1]. Since then, the chaos synchronization quickly became a hot topic. Due to its wide range of applications such as in security communication and oscillator design, chaos synchronization has become an important domain of nonlinear dynamics. With further research, many schemes of chaos synchronization have been developed and widely used in synchronization control of complex network, for example, linear coupling method, feedback approach, adaptive technique, and impulsive control [2-7]. Various kinds of synchronization behaviors have been revealed, such as the complete synchronization, generalized synchronization, phase synchronization, and lag synchronization [8-11].

Recently, generalized projective synchronization (GPS) received extensive attention [12-14]. In [12], authors use the auxiliary system approach to study generalized synchronization and obtain some less restrictive criteria to guarantee the GPS between 
the drive-response systems. In [13], authors researched the projective synchronization (PS) of neural networks with mixed time-varying delays and parameter mismatch. A new weak projective synchronization scheme is proposed to ensure that coupled neural networks are in a state of synchronization with an error level, and several criteria are derived. In [14] authors introduced a generalized projective synchronization method for achieving the different variables of drive-response system synchronized up to different scaling factors. However, the studies mentioned above did not consider the case of chaotic systems with noise disturbance. In fact, noise ubiquitous almost in any real system and many practical systems are very sensitive to parameters' disturbance [15], the synchronization of a concrete model is unavoidably subject to disturbances. So suppression the effect of disturbance in synchronization process is very important in reality. Motivated by this reason, in this paper, we further investigate the GPS of a class of chaotic (or hyperchaotic) systems with uncertain parameters' noise and external disturbances. Via adaptive technique, a novel response system is constructed to synchronize a given chaotic (hyperchaotic) system even if the Lipschitz constant on nonlinear term and the bounds on uncertainty are unknown. Unlike the previous method, the approach proposed in our paper shows high robustness to the parameter noise and external disturbance.

The rest of the paper is organized as follows. In Section 2, the model of our research and preliminaries are introduced. The adaptive scheme for the GPS and noise suppression is presented in Section 3. The numerical simulations with hyperchaotic Lü system are provided to verify the effectiveness of the proposed approach in Section 4. Finally, conclusions are given in Section 5.

\section{Model Description and Preliminaries}

Consider a class of chaotic (or hyperchaotic) systems described by

$$
\dot{x}=A x+f(x)+g(x) v,
$$

where $x=\left(x_{1}, x_{2}, \ldots, x_{n}\right)^{T} \in R^{n}$ is the state vector, $A=\left(a_{i j}\right)_{n \times n}$ is a constant matrix. $f: R^{n} \rightarrow R^{n}$ is a quadratic function vector and each term of $f(x)$ has the form of $x_{i} x_{j}$ or zero. $g: R^{n} \rightarrow R^{n \times r}$ is a linear function matrix. $v \in R^{r}$ is uncertain or unknown parameter vector. Suppose there exists unknown external disturbance which is denoted by $\eta(t) \in R^{n}$, system (2.1) is recast as follows:

$$
\dot{x}=A x+f(x)+g(x) v+\eta(t) .
$$

In fact, many classical chaotic and hyperchaotic systems can be written in the form of (2.1). For instance, the Lorenz system [16], the Chen system [17], the Lü system [18], the unified chaotic system [19], the hyperchaotic Lü system [20], the hyperchaotic Chen system [21], and the hyperchaotic Rössler system [22]. Therefore, the further research on the GPS of such class of chaotic (hyperchaotic) systems is very significant.

In order to construct a response system for the GPS purpose, we introduce some necessary assumptions and lemmas as follows. In the following, norms $\|\cdot\|_{2}$ and $\|\cdot\|_{1}$ of vector $x$ are defined as $\|x\|_{2}=\sqrt{x^{T} x}$ and $\|x\|_{1}=\sum_{i=1}^{n}\left|x_{i}\right|$, respectively. 
Assumption 2.1. There exists nonnegative constants $\delta_{f}$ and $\delta_{g}$ such that:

$$
\|f(x)-f(y)\|_{2} \leq \delta_{f}\|x-y\|_{2}, \quad\|g(x)\|_{2} \leq \delta_{g}\|x\|_{2}
$$

where $x, y$ are time-varying vectors.

Assumption 2.2. The uncertain parameter vector $v$ and external disturbance $\eta(t)$ are norm bounded, that is, there exists positive constants $\delta_{v}$ and $\delta_{\eta}$ satisfying

$$
\|v\|_{2} \leq \delta_{v}, \quad\|\eta(t)\|_{1} \leq \delta_{\eta}
$$

and the disturbance $\eta(t)$ does not destroy the chaotic or hyperchaotic behavior of system (2.1).

Remark 2.3. Let $E$ be a compact subset of $R^{n}$ which contains the chaotic attractor of system (2.1). Obviously, the quadratic function $f$ and linear function $g$ satisfy Assumption 2.1 on $E$.

For the constant matrix $A$, one can easily take $D=\operatorname{diag}\left(d_{1}, d_{2}, \ldots, d_{n}\right)\left(d_{i} \geq 0, i=\right.$ $1,2, \ldots, n)$ such that $A-D$ is Hurwitz matrix. Then there has following lemma.

Lemma 2.4 (see [23]). For the Hurwitz matrix $A-D$, there exists symmetry positive definite matrixes $P$ and $Q$ which satisfy the Lyapunov equation:

$$
(A-D)^{T} P+P(A-D)=-Q
$$

\section{Approach for the GPS and Noise Suppression}

Now we construct a response system to synchronize the system (2.2) in a drive-response framework. Take the system (2.2) as drive system, a response system is constructed as follows:

$$
\dot{\hat{x}}=A \widehat{x}+\alpha^{-1} f(\widehat{x})+g(\widehat{x}) \widehat{v}+u,
$$

where $\hat{x} \in R^{n}$ is the state vector of the response system, $\alpha$ is a nonzero constant, $\widehat{v}$ is the estimation of uncertain parameter vector $v$, and $u$ is the control input.

We say that systems (2.2) and (3.1) achieve GPS if $\lim _{t \rightarrow+\infty}\|\alpha x-\widehat{x}\|_{2}=0$, where $\alpha$ is called the scaling factor. So the synchronous error vector is defined as $e=\alpha x-\widehat{x}$.

If the control input in (3.1) is taken as

$$
u=D e+\widehat{r} \operatorname{sgn}(P e)+\widehat{k} P e,
$$


where $\operatorname{sgn}(\cdot)$ denotes a symbolic function of $(\cdot)$, moreover, it denotes that each component takes the symbolic function when $(\cdot)$ is a vector. $\widehat{r}$ and $\widehat{k}$ are adaptive variables to be designed. The matrixes $P$ and $D$ satisfy (2.5). Then one can obtain the error system as follows:

$$
\begin{aligned}
\dot{e} & =(A-D) e+\alpha^{-1}\left(\alpha^{2} f(x)-f(\widehat{x})\right)+(\alpha g(x) v-g(\widehat{x}) \widehat{v})+\alpha \eta-\widehat{r} \operatorname{sgn}(P e)-\widehat{k} P e \\
& =(A-D) e+\alpha^{-1}(f(\alpha x)-f(\widehat{x}))+(g(\alpha x)-g(\widehat{x})) v+g(\widehat{x})(v-\widehat{v})+\alpha \eta-\widehat{r} \operatorname{sgn}(P e)-\widehat{k} P e \\
& =(A-D) e+\alpha^{-1}(f(\alpha x)-f(\widehat{x}))+g(e) v+g(\widehat{x})(v-\widehat{v})+\alpha \eta-\widehat{r} \operatorname{sgn}(P e)-\widehat{k} P e .
\end{aligned}
$$

Theorem 3.1. Suppose that Assumptions 2.1 and 2.2 hold. Take the control input as (3.2) and adaptive laws as follows:

$$
\begin{gathered}
\dot{\hat{v}}=l_{v}[g(\widehat{x})]^{T} P e, \\
\dot{\hat{r}}=l_{r}(P e)^{T} \operatorname{sgn}(P e)=l_{r}\|P e\|_{1}, \quad \dot{\hat{k}}=l_{k} e^{T} P P e=l_{k}\|P e\|_{2}^{2}
\end{gathered}
$$

where $l_{v}, l_{r}$, and $l_{k}$ are positive constants. Then systems (2.2) and (3.1) can achieve the GPS.

Proof. Choose the Lyapunov function as

$$
V(e, \widehat{v}, \widehat{r}, \widehat{k})=e^{T} P e+\frac{1}{l_{v}}(v-\widehat{v})^{T}(v-\widehat{v})+\frac{1}{l_{r}}(r-\widehat{r})^{2}+\frac{1}{l_{k}}(k-\widehat{k})^{2}
$$

where $r, k$ are the adaptive constants. Obviously, $V$ is positive definite.

Its time derivative along the trajectories of (3.3) (3.5) is given by

$$
\begin{aligned}
\dot{V}(e, \widehat{v}, \widehat{r}, \widehat{k})= & e^{T}\left((A-D)^{T} P+P(A-D)\right) e+2 \alpha^{-1} e^{T} P(f(\alpha x)-f(\widehat{x})) \\
& +2 e^{T} P g(e) v+2 e^{T} P g(\widehat{x})(v-\widehat{v})+2 \alpha e^{T} P \eta-2 \widehat{r} e^{T} P \operatorname{sgn}(P e) \\
& -2 \widehat{k} e^{T} P P e-\frac{2}{l_{v}} \dot{\hat{v}}^{T}(v-\widehat{v})-\frac{2}{l_{r}}(r-\widehat{r}) \dot{\widehat{r}}-\frac{2}{l_{k}}(k-\widehat{k}) \hat{\hat{k}} \\
= & -e^{T} Q e+2 \alpha^{-1} e^{T} P(f(\alpha x)-f(\widehat{x}))+2 e^{T} P g(e) v \\
& +2 \alpha e^{T} P \eta-2 r e^{T} P \operatorname{sgn}(P e)-2 k e^{T} P P e \\
\leq & -e^{T} Q e+2\left|\alpha^{-1}\right| \cdot\left\|e^{T} P\right\|_{2} \cdot\|f(\alpha x)-f(\widehat{x})\|_{2}+2\left\|e^{T} P\right\|_{2} \cdot\|g(e)\|_{2} \cdot\|v\|_{2} \\
& +2|\alpha| \cdot\left\|e^{T} P\right\|_{1} \cdot\|\eta\|_{1}-2 r\|P e\|_{1}-2 k\|P e\|_{2}^{2} \\
\leq & -e^{T} Q e+2 \delta_{f}\left|\alpha^{-1}\right| \cdot\left\|e^{T} P\right\|_{2} \cdot\|e\|_{2}+2 \delta_{g} \delta_{v}\|P e\|_{2} \cdot\|e\|_{2}+2 \delta_{\eta}|\alpha|\left\|e^{T} P\right\|_{1} \\
& -2 r\|P e\|_{1}-2 k\|P e\|_{2}^{2}
\end{aligned}
$$


Journal of Applied Mathematics

$$
\begin{aligned}
\leq & -e^{T} Q e+\left(\frac{\alpha^{-2} \delta_{f}^{2}}{\varepsilon_{1}}\|P e\|_{2}^{2}+\varepsilon_{1}\|e\|_{2}^{2}\right)+\left(\frac{\delta_{g}^{2} \delta_{v}^{2}}{\varepsilon_{2}}\|P e\|_{2}^{2}+\varepsilon_{2}\|e\|_{2}^{2}\right) \\
& -2 k\|P e\|_{2}^{2}+2\left(|\alpha| \delta_{\eta}-r\right)\|P e\|_{1} \\
= & -e^{T}\left(Q-\varepsilon_{1} I_{n}-\varepsilon_{2} I_{n}\right) e+\left(\frac{\alpha^{-2} \delta_{f}^{2}}{\varepsilon_{1}}+\frac{\delta_{g}^{2} \delta_{v}^{2}}{\varepsilon_{2}}-2 k\right)\|P e\|_{2}^{2} \\
& +2\left(|\alpha| \delta_{\eta}-r\right)\|P e\|_{1},
\end{aligned}
$$

where $\varepsilon_{1}$ and $\varepsilon_{2}$ are arbitrary small positive constants, $I_{n}$ denotes a $n$-order identity matrix.

One can take

$$
k=\frac{\alpha^{-2} \delta_{f}^{2}}{2 \varepsilon_{1}}+\frac{\delta_{g}^{2} \delta_{v}^{2}}{2 \varepsilon_{2}}, \quad r=|\alpha| \delta_{\eta},
$$

then there has

$$
\dot{V}(e, \widehat{v}, \widehat{r}, \widehat{k}) \leq-e^{T}\left(Q-\varepsilon_{1} I_{n}-\varepsilon_{2} I_{n}\right) e
$$

Denote $\widetilde{Q}=Q-\varepsilon_{1} I_{n}-\varepsilon_{2} I_{n}$. We can choose $\varepsilon_{1}$ and $\varepsilon_{2}$ small enough such that $\widetilde{Q}$ is positive definite. Then $\dot{V}$ is seminegative definite. Whence system (3.3) is Lyapunov stable, which implies that $e \in L_{\infty}$.

Integration (3.9),

$$
V(e, \widehat{v}, \widehat{r}, \widehat{k}) \leq V(e(0), \widehat{v}(0), \widehat{r}(0), \widehat{k}(0))-\lambda_{\min }(\widetilde{Q}) \int_{0}^{t} e^{T} e d \tau
$$

then $e \in L_{2}$. From (3.3), we have $\dot{e} \in L_{\infty}$. By Barbalat's lemma [23], we have $\lim _{t \rightarrow+\infty}\|e\|_{2}=0$, that is, $\lim _{t \rightarrow+\infty}\|\alpha x-\widehat{x}\|_{2}=0$ for any initial values $x(0), \widehat{x}(0) \in E$. Now the proof is completed.

Corollary 3.2. If parameters in system (2.1) are determined, the drive-response systems are recast as

$$
\begin{aligned}
& \dot{x}=A x+f(x)+\eta(t), \\
& \dot{\hat{x}}=A \hat{x}+\alpha^{-1} f(\widehat{x})+u .
\end{aligned}
$$

Take the control input as (3.2) and adaptive laws as (3.4) and (3.5), then system (3.11) can reach the GPS. 


\section{Numerical Simulations}

In this section, the proposed approach for the GPS is illustrated by the hyperchaotic Lü system [16] which is described by

$$
\begin{aligned}
& \dot{x}_{1}=a\left(x_{2}-x_{1}\right)+x_{4}, \\
& \dot{x}_{2}=-x_{1} x_{3}+c x_{2}, \\
& \dot{x}_{3}=x_{1} x_{2}-b x_{3}, \\
& \dot{x}_{4}=x_{1} x_{3}+d x_{4} .
\end{aligned}
$$

It is hyperchaotic when $a=36, b=3, c=20$, and $d=1$. Here we suppose that $c$, $d$ are unknown parameters. In the numerical simulation, we take $\eta(t)$ which contains the parameter perturbation and the system noise as follows:

$$
\eta(t)=\left[0.1\left(x_{2}-x_{1}\right) \sin t, 0.1 \sin (2 t), 0.1 x_{3} \sin (3 t), \sin (4 t)\right]^{T} .
$$

Then the drive system and controlled response system are described as follows:

$$
\begin{gathered}
\dot{x}=A x+f(x)+g(x) v+\eta(t), \\
\dot{\hat{x}}=A \widehat{x}+\alpha^{-1} f(\widehat{x})+g(\widehat{x}) \widehat{v}+D e+\widehat{r} \operatorname{sgn}(P e)+\widehat{k} P e
\end{gathered}
$$

where $x=\left[x_{1}, x_{2}, x_{3}, x_{4}\right]^{T}, \widehat{x}=\left[\widehat{x}_{1}, \widehat{x}_{2}, \widehat{x}_{3}, \widehat{x}_{4}\right]^{T}, v=[c, d]^{T}, \widehat{v}=[\widehat{c}, \widehat{d}]^{T}$,

$$
\begin{gathered}
e=\left[\alpha x_{1}-\widehat{x}_{1}, \alpha x_{2}-\widehat{x}_{2}, \alpha x_{3}-\widehat{x}_{3}, \alpha x_{4}-\widehat{x}_{4}\right]^{T}, \\
A=\left[\begin{array}{cccc}
-36 & 36 & 0 & 1 \\
0 & 0 & 0 & 0 \\
0 & 0 & -3 & 0 \\
0 & 0 & 0 & 0
\end{array}\right], \quad f(x)=\left[\begin{array}{c}
0 \\
-x_{1} x_{3} \\
x_{1} x_{2} \\
x_{1} x_{3}
\end{array}\right], \quad g(x)=\left[\begin{array}{cc}
0 & 0 \\
x_{2} & 0 \\
0 & 0 \\
0 & x_{4}
\end{array}\right] .
\end{gathered}
$$

We can take $D=\operatorname{diag}(0,1,0,1)$ and

$$
P=\left[\begin{array}{cccc}
2 & -1.5 & 0 & 0 \\
-1.5 & 4 & -1 & 0 \\
0 & -1 & 2 & 0 \\
0 & 0 & 0 & 1
\end{array}\right]
$$

Denote

$$
\begin{gathered}
\zeta_{1}=2\left(\alpha x_{1}-\widehat{x}_{1}\right)-1.5\left(\alpha x_{2}-\widehat{x}_{2}\right), \quad \zeta_{2}=-1.5\left(\alpha x_{1}-\widehat{x}_{1}\right)+4\left(\alpha x_{2}-\widehat{x}_{2}\right)-\left(\alpha x_{3}-\widehat{x}_{3}\right), \\
\zeta_{3}=-\left(\alpha x_{2}-\widehat{x}_{2}\right)+2\left(\alpha x_{3}-\widehat{x}_{3}\right), \quad \zeta_{4}=\alpha x_{4}-\widehat{x}_{4} .
\end{gathered}
$$


Then the adaptive laws are

$$
\begin{gathered}
\dot{\hat{c}}=l_{c} \zeta_{2} \widehat{x}_{2}, \quad \dot{\hat{d}}=l_{d} \zeta_{4} \widehat{x}_{4} \\
\dot{\hat{r}}=l_{r}\left(\left|\zeta_{1}\right|+\left|\zeta_{2}\right|+\left|\zeta_{3}\right|+\left|\zeta_{4}\right|\right), \quad \dot{\hat{k}}=l_{k}\left(\zeta_{1}^{2}+\zeta_{2}^{2}+\zeta_{3}^{2}+\zeta_{4}^{2}\right) .
\end{gathered}
$$

By Theorem, systems (4.3) and (4.4) will achieve GPS with scaling factor $\alpha(\alpha \neq 0)$. Figure 1 is the numerical simulation result with the scaling factor $\alpha=-1$. Figures 1 (a) and 1(b) display the comparison of the attractors of the drive-response systems in $R^{3}$ and evolution of the synchronous errors, respectively.

In the numerical simulation, all the differential equations are solved by the fourthorder Runge-Kutta method. The initial values are $x(0)=[1,5,-5,10]^{T}, \widehat{x}(0)=[2,0,-5,-1]^{T}$, $[\widehat{c}(0), \widehat{d}(0)]=[1,1], \widehat{r}(0)=2$, and $\widehat{k}(0)=2$. Take $l_{c}=l_{d}=l_{r}=l_{k}=1$. Note that, here, the adaptive strength $l_{c}, l_{d}, l_{r}$, and $l_{k}$ can be chosen other values, which can control the speed of convergence of the synchronous errors.

As parameters in systems (4.3) and (4.4) are known (where $g$ is zero matrix), by Corollary, the GPS also can be obtained with adaptive law (4.9). Figure 2 displays the simulation result. Figures 2(a) and 2(b) display the same as Figures 1(a) and 1(b) but the system parameters are known and $\alpha=2$. Here, in order to make the image clear, we have translated response system states by +20 units in Figure 2(a).

From Figures 1 and 2, one can see that the synchronous errors converge to zero. That is, the response system (4.4) quickly synchronized to drive system (4.3), the results are not affected by the noise.

Remark 4.1. From Theorem and the numerical simulation results, we notice that the adaptive laws are independent on $\delta_{f}, \delta_{g}, \delta_{v}$, and $\delta_{\eta}$, that is to say, the GPS can be achieved even if the Lipschitz constant and the bounds on uncertainties are unknown. Therefore the approach proposed in our paper shows high robustness to the parameter mismatch and external disturbance.

Remark 4.2. The matrixes $D$ and $P$ in the control input are independent on the variability of the scaling factor, so one can conveniently adjust the scaling factor to any desired scale to realize the GPS. Specially, we can obtain the completely synchronization and antisynchronization by taking $\alpha=1$ and $\alpha=-1$, respectively.

\section{Conclusion}

In this paper, we have proposed a novel robust adaptive scheme for achieving the GPS of a class of chaotic (or hyperchaotic) systems. The control input and adaptive laws in the response system is designed so as to successfully achieve the GPS. One can conveniently adjust the scaling factor to realize the GPS in any desired scale, including completely synchronization, antisynchronization, and general projective synchronization. The numerical simulation shows that the GPS cannot be destroyed by the noise disturbances, that is, the proposed GPS scheme is high robust and is of great significance for improving chaotic secure communications capability. 


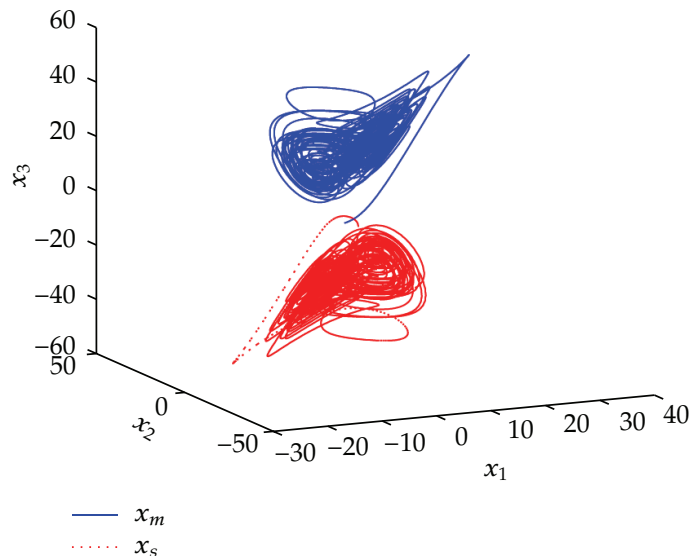

(a)

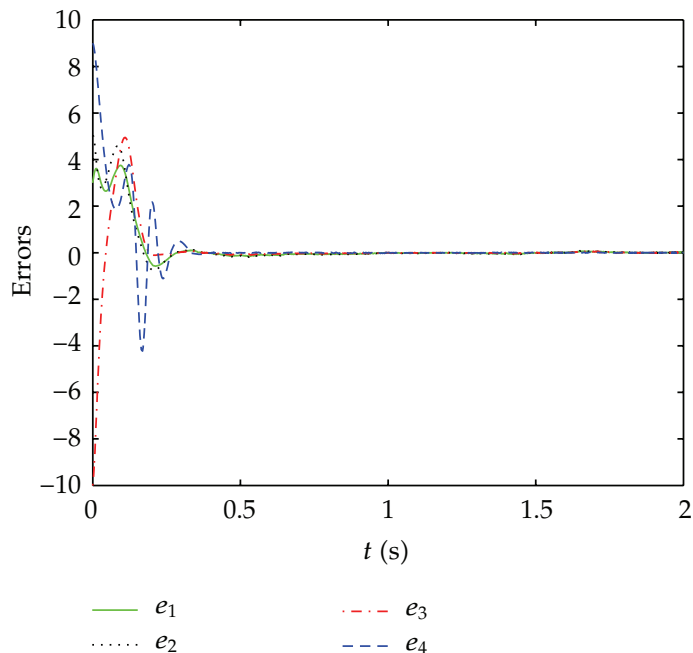

(b)

Figure 1: The GPS between systems (4.3) and (4.4) with uncertain parameters as $\alpha=-1$.

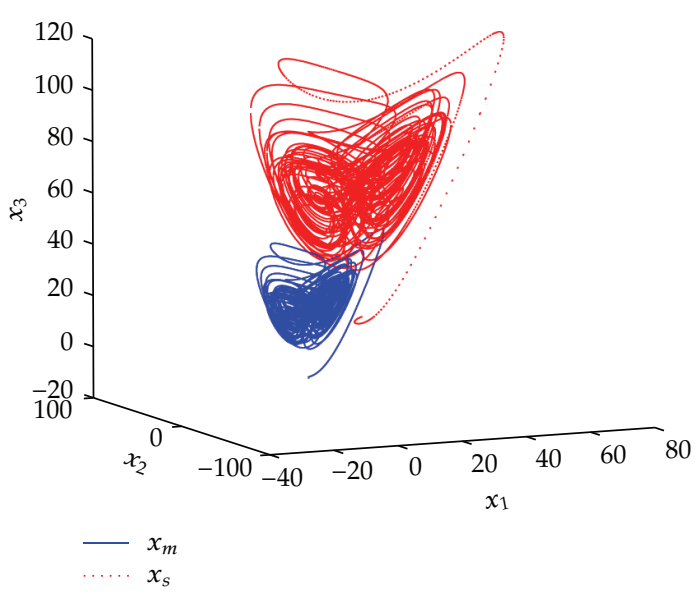

(a)

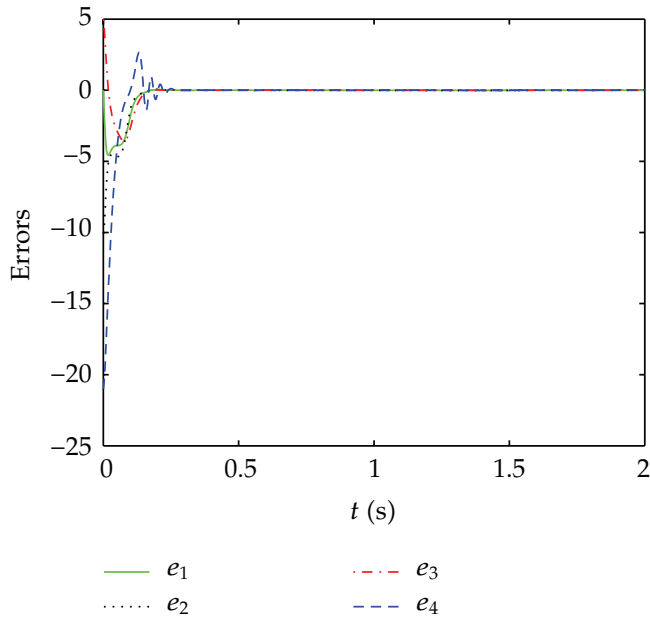

(b)

Figure 2: The GPS between systems (4.3) and (4.4) with certain parameters as $\alpha=2$.

\section{Acknowledgments}

This work was jointly supported by the National Natural Science Foundation of China under Grant no. 61164020, 61004101, and the Natural Science Foundation of Guangxi under Grant no. 2011 GXNSFA018147, Guangxi Key Laboratory of Spatial Information and Geomatics (no. 1103108-24). 


\section{References}

[1] L. M. Pecora and T. L. Carroll, "Synchronization in chaotic systems," Physical Review Letters, vol. 64, no. 8, pp. 821-824, 1990.

[2] G. Chen and J. Lü, Dynamics of the Lorenz System Family: Analysis, Control and Synchronization, Science Press, Beijing, China, 2003.

[3] Z. Jia and J. Lu, "Nonlinearly state feedback and adaptive synchronization of hyperchaotic Lü system," Systems Engineering E Electronics, vol. 29, pp. 598-600, 2007.

[4] J. Cao, D. W. C. Ho, and Y. Yang, "Projective synchronization of a class of delayed chaotic systems via impulsive control," Physics Letters A, vol. 373, no. 35, pp. 3128-3133, 2009.

[5] P. Li and J. Cao, "Stabilisation and synchronisation of chaotic systems via hybrid control," IET Control Theory and Applications, vol. 1, no. 3, pp. 795-801, 2007.

[6] J. Zhou, J. Lu, and J. Lü, "Adaptive synchronization of an uncertain complex dynamical network," IEEE Transactions on Automatic Control, vol. 51, no. 4, pp. 652-656, 2006.

[7] J. Lü and G. Chen, "A time-varying complex dynamical network model and its controlled synchronization criteria," IEEE Transactions on Automatic Control, vol. 50, no. 6, pp. 841-846, 2005.

[8] N. F. Rulkov, M. M. Sushchik, L. S. Tsimring, and H. D. I. Abarbanel, "Generalized synchronization of chaos in directionally coupled chaotic systems," Physical Review E, vol. 51, no. 2, pp. 980-994, 1995.

[9] M. G. Rosenblum, A. S. Pikovsky, and J. Kurths, "Phase synchronization of chaotic oscillators," Physical Review Letters, vol. 76, no. 11, pp. 1804-1807, 1996.

[10] M. G. Rosenblum, A. S. Pikovsky, and J. Kurths, "From phase to lag synchronization in coupled chaotic oscillators," Physical Review Letters, vol. 78, pp. 4193-4196, 1997.

[11] J. Lü, X. Yu, G. Chen, and D. Cheng, "Characterizing the synchronizability of small-world dynamical networks," IEEE Transactions on Circuits and Systems I, vol. 51, no. 4, pp. 787-796, 2004.

[12] W. He and J. Cao, "Generalized synchronization of chaotic systems: an auxiliary system approach via matrix measure," Chaos, vol. 19, no. 1, Article ID 013118, 10 pages, 2009.

[13] S. Chen and J. Cao, "Projective synchronization of neural networks with mixed time-varying delays and parameter mismatch," Nonlinear Dynamics, vol. 67, pp. 1397-1406, 2012.

[14] Z. Jia, J. A. Lu, G. M. Deng, and Q. J. Zhang, "Generalized projective synchronization of a class of chaotic (hyperchaotic) systems with uncertain parameters," Chinese Physics, vol. 16, no. 5, pp. 12461251, 2007.

[15] P. Wang, J. Lu, and M. Ogorzalek, “Global relative parameter sensitivities of the feed-forward loops in genetic networks," Neurocomputing, vol. 78, no. 1, pp. 155-165, 2012.

[16] E. N. Lorenz, "Deterministic non-periods flows," Journal of the Atmospheric Sciences, vol. 20, pp. 130141, 1963.

[17] G. Chen and T. Ueta, "Yet another chaotic attractor," International Journal of Bifurcation and Chaos, vol. 9, no. 7, pp. 1465-1466, 1999.

[18] J. Lü and G. Chen, "A new chaotic attractor coined," International Journal of Bifurcation and Chaos, vol. 12, no. 3, pp. 659-661, 2002.

[19] J. Lü, G. Chen, D. Cheng, and S. Celikovsky, "Bridge the gap between the Lorenz system and the Chen system," International Journal of Bifurcation and Chaos, vol. 12, no. 12, pp. 2917-2926, 2002.

[20] A. Chen, J. Lu, J. Lü, and S. Yu, "Generating hyperchaotic Lü attractor via state feedback control," Physica A, vol. 364, pp. 103-110, 2006.

[21] Y. Li, W. K. S. Tang, and G. Chen, "Generating hyperchaos via state feedback control," International Journal of Bifurcation and Chaos, vol. 15, no. 10, pp. 3367-3375, 2005.

[22] O. E. Rössler, “An equation for hyperchaos," Physics Letters A, vol. 71, no. 2-3, pp. 155-157, 1979.

[23] H. K. Khalil, Nonlinear Systems, Prentice Hall, New Jersey, NJ, USA, 3rd edition, 2002. 


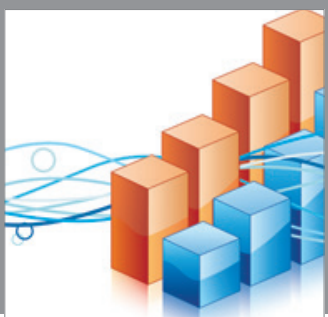

Advances in

Operations Research

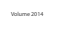

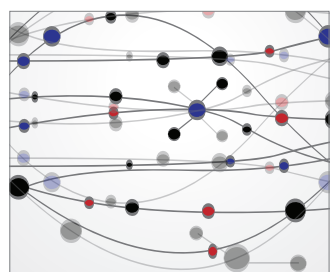

\section{The Scientific} World Journal
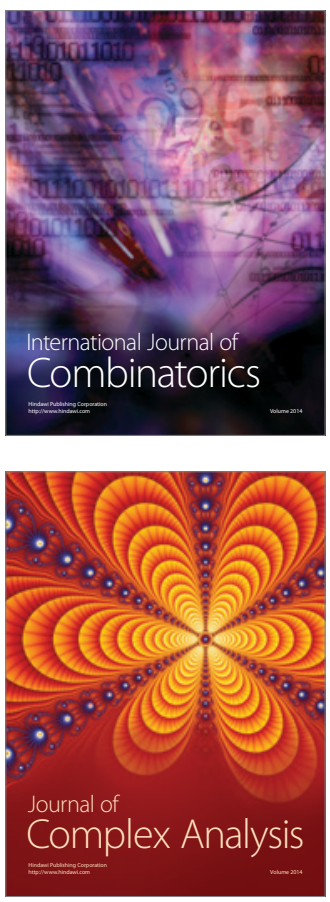

International Journal of

Mathematics and

Mathematical

Sciences
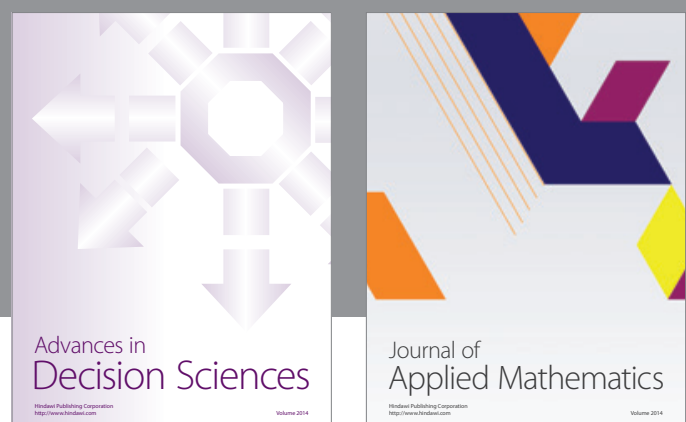

Journal of

Applied Mathematics
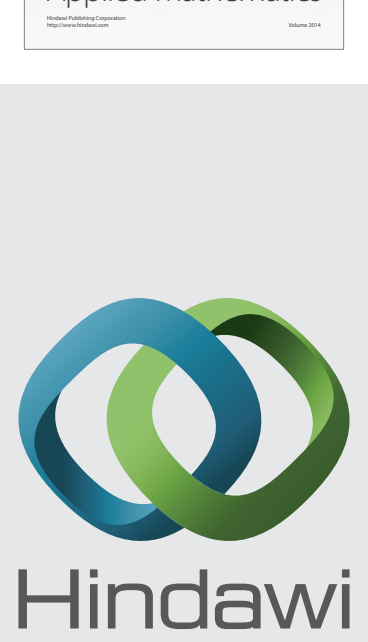

Submit your manuscripts at http://www.hindawi.com
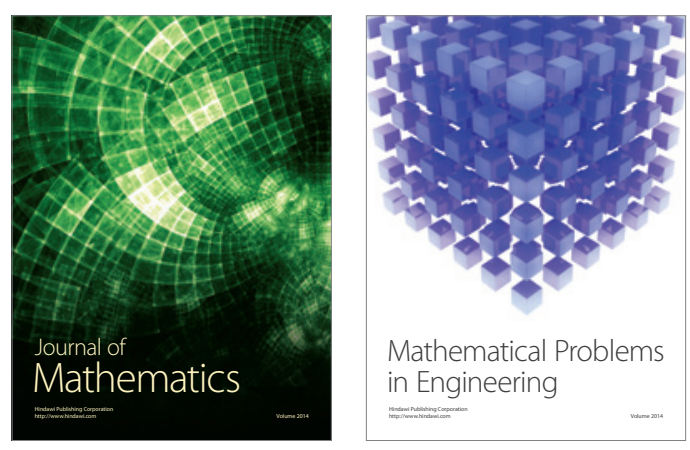

Mathematical Problems in Engineering
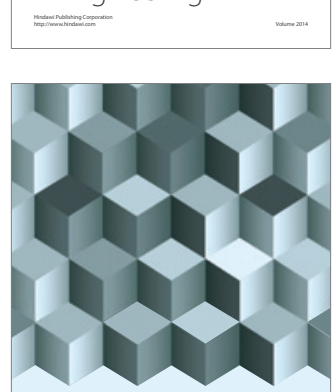

Journal of

Function Spaces
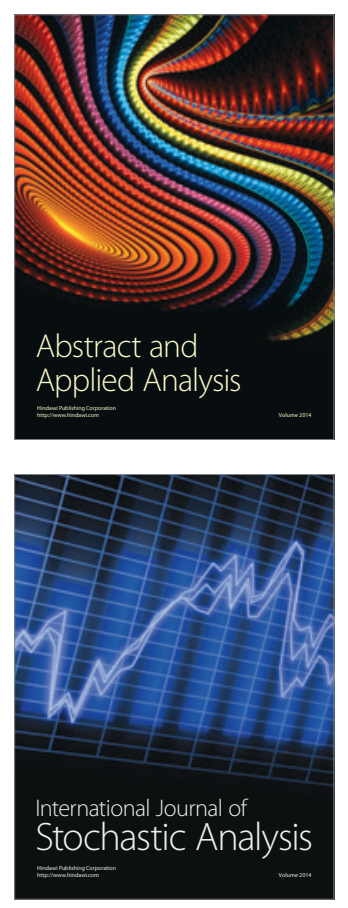

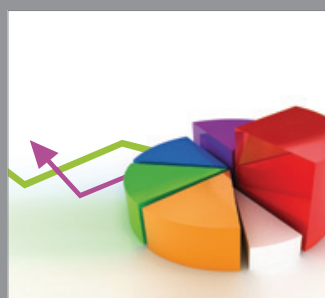

ournal of

Probability and Statistics

Promensencen
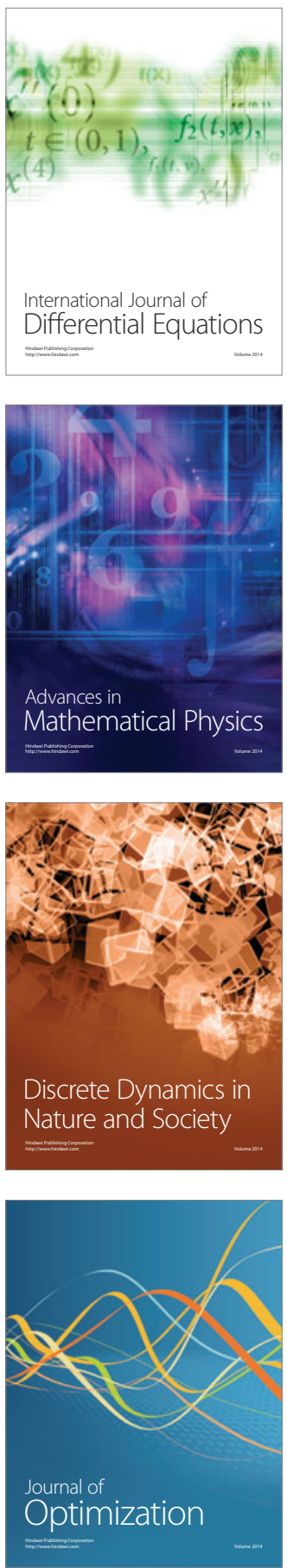